\title{
SKA3 Serves as a Biomarker for Poor Prognosis in Kidney Renal Papillary Cell Carcinoma
}

\author{
Dechao Feng (iD* \\ Facai Zhang* \\ Ling Liu* \\ Qiao Xiong \\ Hang Xu \\ Wuran Wei \\ Zhenghua Liu \\ Lu Yang (D)
}

Department of Urology, Institute of Urology, West China Hospital, Sichuan University, Chengdu, 6I004I, People's Republic of China

*These authors contributed equally to this work
Correspondence: Zhenghua Liu; Lu Yang Department of Urology, Institute of Urology, West China Hospital, Sichuan University, 37 Guoxue Xiang, Chengdu, 61004I, Sichuan, People's Republic of

China

Tel $+8628-8542-2444$

Fax +86 28-8542245I

Email zhliu@scu.edu.cn; wycleflue@163. com
Background: There is a surprising paucity of studies investigating the potential mechanism of SKA3 in the progression and prognosis of kidney renal papillary cell carcinoma (KIRP). Methods: We used TCGA and other databases to analyze the expression, clinical value, and potential mechanisms of SKA3 in KIRP patients. We also explored therapeutic agents for KIRP through GSCALite.

Results: SKA3 mRNA expression was significantly upregulated and the area under the curve was 0.792 (95\% CI 0.727-0.856). Increased SKA3 expression was related to shorter overall survival, disease-specific survival and progression-free survival. Hub genes in protein-protein interactions were $C D K 1, C D C 20, C C N B 1, C C N A 2, B U B 1, A U R K B, B U B 1 B$, $P L K 1, C C N B 2$, and $M A D 2 L 1$, which were differentially expressed and also associated with KIRP prognosis. Gene-set enrichment analysis indicated that E2F targets, epithelialmesenchymal transition, glycolysis, the WNT signaling pathway, and other pathways were highly enriched upon SKA3 upregulation. Gene-set variation analysis of SKA3 and its ten hub genes showed that the significant correlation of cancer-related pathways included the cell cycle, DNA damage, hormone androgen receptor, hormone estrogen receptor, PI3K/Akt, and Ras/MAPK. In addition, we found that MEK inhibitors, ie, trametinib, selumetinib, PD0325901, and RDEA119, may be feasible targeting agents for KIRP patients.

Conclusion: SKA3 might contribute to poor prognosis of KIRP through cell cycle, DNA damage, hormone androgen receptor, hormone estrogen receptor, PI3K/Akt, and RAS/ MAPK. SKA3 potentially serves as a prognostic biomarker and target for KIRP.

Keywords: spindle and kinetochore-associated complex subunit 3, kidney renal papillary cell carcinoma, biomarker, enrichment analysis, targeted therapy

\section{Introduction}

Incidence of renal cell carcinoma (RCC) varies widely, with an estimated 431,288 new cases and 179,368 deaths worldwide in $2020 .{ }^{1}$ Despite improvements in the management of RCC, it remains one of the most fatal urological malignancies. ${ }^{2}$ Clear-cell carcinoma is the most frequent histological subtype, while kidney renal papillary cell carcinoma (KIRP) accounts for $10 \%-20 \%{ }^{3}$ Compared to RCC, the clinical behaviors of KIRP are more changeable in consist with epidemiology. KIRP occurs sporadically, and only around $4 \%$ of patients have a hereditary link. ${ }^{4}$ The clinical manifestations of KIRP and RCC are similar, and radical or partial nephrectomy remains the mainstream treatment for the localized stage, with almost $40 \%$ recurrence. ${ }^{3}$ For some patients at the local advanced stage or metastasis, several agents, including anti-VEGF drugs and mTOR inhibitors, provide limited efficacy. ${ }^{5,6}$ A considerable number of studies have focused on molecular 
investigation and clinical trials of RCC, whereas there is a notable paucity of high-quality research on molecular profiling and treatment of KIRP seeking to identify novel targets for precisely individualized therapy.

SKA3 regulates microtubule attachment to kinetochores during mitosis, and may be essential for normal chromosome segregation and cell division. ${ }^{7}$ Studies have indicated that overexpression of SKA3 is associated with disease progression and prognosis of some malignant tumors. ${ }^{8-11}$ To date, however, there has been little published information on the role of SKA3 in KIRP. In this study, we sought for the first time to explore the relationship between differential expression of SKA3 and clinical values in KIRP, with insights into potential mechanisms and possible treatments via bioinformatic analysis.

\section{Methods}

\section{Data Extraction}

We acquired data from the University of California, Santa Cruz Xena database (https://xenabrowser.net/datapages), ${ }^{12}$ which includes the Cancer Genome Atlas (TCGA) and Genotype-Tissue Expression databases. The data were processed through Toil. ${ }^{13}$ Prognostic data were obtained from Liu et al. ${ }^{14}$ Transcripts per million reads were used, and $\log _{2}$ conversion was performed for all data in standardization.

\section{Analysis of Differential Expression and Clinical Values}

The data were used to analyze SKA3 mRNA expression in pan-cancer and KIRP. We assessed diagnostic and survival values of SKA3 expression through area under the curve and survival analysis. Overall survival (OS), diseasespecific survival (DSS) and progression-free survival (PFS) were used as prognostic factors.

\section{Biological Functional Analysis}

Coexpressed genes of SKA3 were identified with Pearson correlation coefficients $(R>0.4$ and $P<0.001)$. Gene Ontology (GO) and Kyoto Encyclopedia of Genes and Genomes (KEGG) analysis was performed on coexpressed genes with R clusterProfiler to explore possible biological functions and signaling pathways affected by SKA3. ${ }^{15} \mathrm{GO}$ analysis included biological process, cell composition, and molecular function, with $P<0.05$ taken as statistically significant). TCGA data were analyzed with gene-set enrichment analysis. The threshold for significant enrichment was a false-discovery rate $<0.25$ and adjusted $P<0.05$. A protein-protein interaction (PPI) network of SKA3-coexpressed genes was identified through the STRING database (https://www.string-db.org), and high confidence was set as 0.7. The network obtained was imported into Cytoscape 3.7.2, ${ }^{16}$ and the first ten genes were defined as hub genes using the CytoHubba plug-in and ranked by degree. We further analyzed cancer-related pathways of SKA3 and the ten hub genes through gene-set variation analysis (GSVA) via GSCALite. ${ }^{17}$

\section{Analysis Using CBioportal and GSCALite}

The CBioportal database (www.cbioportal.org) ${ }^{18,19}$ was used to explore the association between SKA3 mRNA expression (RNA Seq V2 RSEM) and hypoxia-related parameters. We used the GSCALite database to further analyze mutation information, immunoinfiltration of SKA3, and coexpressed genes. ${ }^{17}$ We also explored the Genomics of Drug Sensitivity in Cancer database and Cancer Therapeutics Response Portal for drug sensitivity and expression correlations of the top ten hub genes coexpressed with SKA3 through GSCALite. ${ }^{17}$

\section{Statistical Analysis}

R 3.6.3 software was used for statistical analysis with the packages ggplot2, pROC, survival, survminer, clusterProfiler, and rms. The chi-square test was used to assess differences between categorical variables and paired or unpaired $t$-tests used for continuous variables. The Wilcoxon rank-sum test was used if Shapiro-Wilk normality testing yielded significance. Survival was analyzed using Kaplan-Meier curves and log-rank tests. All tests were two-sided. $P<0.05$ was considered statistically significant

\section{Results}

\section{Basic Clinical Features and Values}

A total of 289 tumor patients in TCGA were enrolled. Median SKA3 expression was used to define the high and low groups. The expression of SKA3 was related to clinical $\mathrm{T}$ stage, $\mathrm{N}$ stage, $\mathrm{M}$ stage, sex, race, and age. Table 1 presents the relationship of SKA3 expression with basic clinical features. SKA expression was differentially expressed through paired and unpaired analysis of pan-cancer and KIRP level (Figure 1A-D). The expression of SKA3 showed an upward trend in stages (Figure 1E). 
The area under the curve was 0.851 (95\% CI 0.796-0.906, Figure 1F). The top ten positively SKA3-related genes were NUF2, HJURP, CDK1, KIFC1, NCAPG, SKA1, CEP55, TTK, DLGAP5, and NDC80, while negatively SKA3-related genes were $L D H D, C K B, O G D H L, M Y L 3$, PHYHD1, ZNF385B, CHCHD10, C3orf18, C16orf86, and RYR2 (Figure 1G). Increased SKA3 expression was significantly related to shorter OS (HR 2.31, 95\% CI 1.28 4.18; Figure 1H), DSS (HR 6.50, 95\% CI 3.14-13.64; Figure 1I) and PFS (HR 3.54, 95\% CI 2.10-5.97; Figure 1J). For advanced-stage (III and IV) patients, Caucasian ethnicity, BMI $\leq 30$, complete remission, M0 stage, T3-T4 stage, no smoking, and left side, higher expression of SKA3 was significantly associated with poorer OS (Figure $2 \mathrm{~A}-\mathrm{H}$ ). For patients aged $<60$ years, $\mathrm{BMI} \leq 30$, and left side, higher expression of SKA3 was significantly associated with poorer DSS (Figure 2I-K). For patients in T1 stage, N0 stage, and left side, higher expression of SKA3 was significantly associated with shorter PFS (Figure 2L-N).

\section{Gene Enrichment Analysis}

GO and KEGG showed that SKA3 was mainly enriched in the cell cycle, DNA replication, RNA transport, ubiquitinmediated proteolysis, p53 signaling pathway, organelle fission, nuclear division, chromosome segregation, and chromosomal region (Figure 3A and B). Gene-set enrichment analysis indicated that highly enriched hallmarks included E2F targets, epithelial-mesenchymal transition, glycolysis, $\mathrm{G}_{2} \mathrm{M}$ checkpoint, and genes downregulated by KRAS activation upon SKA3 upregulation (Figure 3C). Pathways significantly enriched by SKA3 overexpression included PLK1, FOXM1, and Aurora B signaling, with MET activating PTK2 signaling and cell-cycle checkpoints and promoting cell motility, DNA replication, the Wnt signaling pathway, and GPCR-ligand binding (Figure 3D-F). Hub genes in protein-protein interactions were $C D K 1, C D C 20, C C N B 1, C C N A 2, B U B 1, A U R K B$, $B U B 1 B, P L K 1, C C N B 2$, and MAD2L1 (Figure 3G). GSVA of SKA3 and its ten hub genes showed that the tumor group had higher GSVA scores than the normal group $(P<0.001$, Figure $3 \mathrm{H})$. The box plot showed that patients in advanced stages (III and IV) had significantly higher GSVA scores than those in early stages (I and II) in KIRP patients $\left(P=5.73^{-6}\right.$, Figure 3I). Higher GSVA scores were related to greater risk of OS and PFS (Figure 3J). Significantly enriched cancer-related pathways were the cell cycle, DNA damage, hormone androgen receptor, hormone estrogen receptor, PI3K/Akt, and RAS/MAPK (Figure 3K). The ten hub genes were significantly highly expressed in KIRP patients, and their overexpression was significantly associated with poor prognosis (Figure 4).

\section{Analysis of GSCALite and CBioportal}

Figure 5 shows genomic alterations of SKA3 and hub genes from three domains: single-nucleotide variation (SNV; Figure 5A-E), copy-number variation (CNV; Figure 5F-K), and methylation (Figure 5L-N). SKA3 showed no effective mutation in KIRP (Figure 5A), and no survival difference was observed between mutants and wild types (Figure 5E). CNV in KIRP patients included heterozygous, homozygous, amplification, and deletion (Figure 5G). No significant correlation was found with regard to heterozygous or homozygous CNV. Correlations of CNV with mRNA expression were significant for SKA3, MAD2L1, and CCNA2 (Figure 5I). In KIRP patients, significant OS and PFS differences were detected between CNV groups and wild groups, except for $B U B 1$ (Figure 5J). Gene-set CNV also had worse prognosis than wild-type in KIRP patients (Figure 5K). There were methylation differences between tumor and normal samples of genes in KIRP (Figure 5L). Hypomethylation of $A U R K B$ was associated with worse OS in KIRP (Figure $5 \mathrm{M}$ ). In addition, we found there were negative correlations between methylation and mRNA expression for SKA3, MAD2L1, CDC20, CCNA2, and AURKB (Figure $5 \mathrm{~N}$ ). In single-gene analysis, we found no associations between mRNA expression, $\mathrm{CNV}$, methylation of SKA3, or immunoinfiltration (Figure 6A-C). CD8-naïve and $\mathrm{CD}^{+} \mathrm{T}$ cells were positively correlated with GSVA scores, while infiltration scores, $\mathrm{CD}^{+}{ }^{+} \mathrm{T}$ cells, follicular helper $\mathrm{T}$ cells, and dendritic cells were negatively related to GSVA scores (Figure 6D). Compared to wild-type samples, dendritic cells, $\gamma \gamma$ cells, and monocytes were significantly enriched at higher amplification, while Th17, natural killer, $\mathrm{nT}_{\text {reg }}$, and $\mathrm{iT}_{\text {reg }}$ cells were enriched at lower amplification (Figure 6E). There was no significant difference between immunoinfiltration and gene-set SNV group (Figure 6F). With the help of GSCALite, ${ }^{17}$ we found that MEK inhibitors, ie, trametinib, selumetinib, PD0325901, and RDEA119, may be feasible targeted agents in KIRP patients with high expression of SKA3 and coexpressed genes (Figure 6G and $\mathrm{H}$ ). In addition, hypoxia scores (Buffa, Ragnum, and Winter) and MSIsensor score were significantly higher in the SKA3- 
Table I Relationships between SKA3 expression and clinical features in KIRP patients in TCGA database

\begin{tabular}{|c|c|c|c|}
\hline & $\begin{array}{c}\text { Low } \\
\text { expression } \\
(n=\mid 44)\end{array}$ & $\begin{array}{c}\text { High } \\
\text { expression } \\
(n=145)\end{array}$ & $P$ \\
\hline Clinical T stage, n (\%) & & & $<0.001$ \\
\hline TI & 80 (39.8\%) & 59 (29.4\%) & \\
\hline $\mathrm{T} 2$ & $14(7 \%)$ & $12(6 \%)$ & \\
\hline T3 & 7 (3.5\%) & 28 (I3.9\%) & \\
\hline $\mathrm{T} 4$ & 0 & I (0.5\%) & \\
\hline Clinical N stage, $\mathbf{n}(\%)$ & & & $<0.001$ \\
\hline No & $71(46.4 \%)$ & $6 \mathrm{I}(39.9 \%)$ & \\
\hline $\mathrm{NI}$ & $2(1.3 \%)$ & 17 (II.I\%) & \\
\hline N2 & 0 & $2(1.3 \%)$ & \\
\hline Clinical M stage, n (\%) & & & 0.037 \\
\hline Mo & $98(46.9 \%)$ & $102(48.8 \%)$ & \\
\hline MI & I (0.5\%) & $8(3.8 \%)$ & \\
\hline Sex, n (\%) & & & 0.004 \\
\hline Female & 27 (9.3\%) & $50(17.3 \%)$ & \\
\hline Male & $117(40.5 \%)$ & 95 (32.9\%) & \\
\hline Race, n (\%) & & & 0.015 \\
\hline Asian & 0 & $6(2.2 \%)$ & \\
\hline Black or African & 27 (9.9\%) & $34(12.5 \%)$ & \\
\hline American & & & \\
\hline White & 110 (40.4\%) & 95 (34.9\%) & \\
\hline Age (years), n (\%) & & & $<0.001$ \\
\hline$\leq 60$ & $5 \mathrm{I}(\mathrm{I7.8 \% )}$ & $82(28.7 \%)$ & \\
\hline$>60$ & 91 (3I.8\%) & $62(21.7 \%)$ & \\
\hline Weight, n (\%) & & & 0.090 \\
\hline$\leq 80$ & $37(16.9 \%)$ & $46(21 \%)$ & \\
\hline$>80$ & $78(35.6 \%)$ & $58(26.5 \%)$ & \\
\hline Height (cm), n (\%) & & & 0.130 \\
\hline$\leq 170$ & 39 (I8.3\%) & 48 (22.5\%) & \\
\hline$>170$ & 7I (33.3\%) & $55(25.8 \%)$ & \\
\hline BMI, n (\%) & & & 0.351 \\
\hline$\leq 30$ & $74(34.7 \%)$ & $62(29.1 \%)$ & \\
\hline$>30$ & $36(16.9 \%)$ & $4 \mathrm{l}(19.2 \%)$ & \\
\hline Smoker, n (\%) & & & 0.486 \\
\hline No & $55(22.3 \%)$ & $6 \mathrm{I}(24.7 \%)$ & \\
\hline Yes & $69(27.9 \%)$ & $62(25.1 \%)$ & \\
\hline Serum calcium, n (\%) & & & 0.395 \\
\hline Elevated & $2(1.1 \%)$ & $4(2.2 \%)$ & \\
\hline Low & 24 (13.3\%) & 17 (9.4\%) & \\
\hline Normal & $65(36.1 \%)$ & 68 (37.8\%) & \\
\hline Hemoglobin, n (\%) & & & 0.145 \\
\hline Elevated & 0 & I (0.5\%) & \\
\hline Low & $42(20.2 \%)$ & $53(25.5 \%)$ & \\
\hline
\end{tabular}

(Continued)
Table I (Continued).

\begin{tabular}{|l|c|c|c|}
\hline & $\begin{array}{c}\text { Low } \\
\text { expression } \\
(\mathbf{n = 1 4 4 )}\end{array}$ & $\begin{array}{c}\text { High } \\
\text { expression } \\
(\mathbf{n = 1 4 5 )}\end{array}$ & $\mathbf{P}$ \\
\hline Normal & $61(29.3 \%)$ & $51(24.5 \%)$ & \\
\hline $\begin{array}{l}\text { Laterality, n (\%) } \\
\text { Left } \\
\text { Right }\end{array}$ & $76(26.6 \%)$ & $84(29.4 \%)$ & 0.484 \\
$66(23.1 \%)$ & $60(21 \%)$ & \\
\hline
\end{tabular}

Abbreviations: KRIP, kidney renal papillary cell carcinoma; BMI, body-mass index.

altered group than in the unaltered group on CBioportal (Figure 6I-L).

\section{Discussion}

KIRP has been demonstrated to be a heterogeneous disease in response to genomic alterations and clinical behaviors. ${ }^{20}$ One study classified KIRP into two types, with type 1 altered in the MET pathway and type 2 related to activation of the NRF2-antioxidant response element pathway. ${ }^{21}$ However, what is not yet understood is the actual relationship between genome variants and clinical behaviors due to relatively low incidence and lack of solid data. Despite several agents, including mTOR inhibitors and VEGF receptor inhibitors, being possible options in the treatment of advanced and metastatic KIRP, the efficacy is unsatisfactory. ${ }^{5,6}$ Given this, there is an urgent need to uncover new target for diagnosis, treatment, and prognosis of KIRP.

SKA3 is a component of the SKA1 complex, a microtubule-binding subcomplex of the outer kinetochore that is essential for proper chromosome segregation. ${ }^{22,23}$ The SKA complex contributes to the stabilization of the kinetochore-microtubule interface through the network of KNL1, Mis12, and Ndc80. ${ }^{23,24}$ The phosphorylation of SKA3 with CDK1 is essential for kinetochore localization of the SKA complex during mitosis. ${ }^{9,25}$ SKA3 can also integrate BUB3 via competing BUB1/BUBR1, helping to silence spindleassembly checkpoint. ${ }^{22,26,27}$ Both $B U B 1$ and $C D K 1$ were members of the SKA3 hub-gene set, and thus we thought that gene interactions contributed to cell proliferation through regulation of the cell cycle. We also observed enrichment of the p53 signaling pathway upon SKA3 upregulation in KIRP patients. The TP53 gene is well known as a tumor-suppressor gene that plays a pivotal role in regulation of the cell cycle and DNA restoration. ${ }^{28-30}$ Mutated TP53 can lead to 

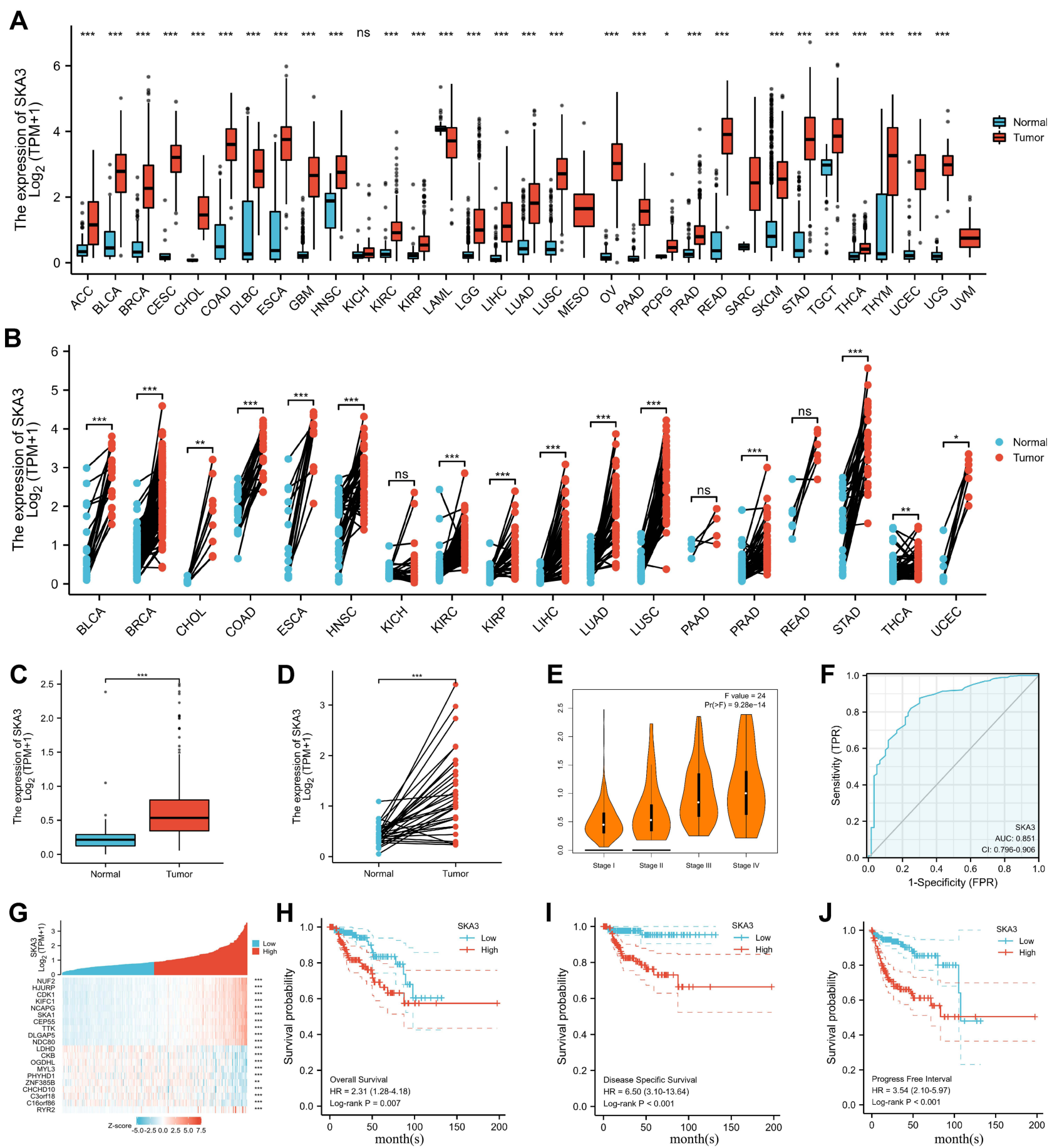

Figure I Clinical values, coexpressed genes, and expression of SKA3 in KIRP patients. (A) SKA3 expression in unpaired samples in pan-cancer; (B) SKA3 expression in paired samples in pan-cancer; (C) SKA3 expression in unpaired sample in KIRP; (D) SKA3 expression in paired sample in KIRP; (E) SKA3 expression among stages in KIRP; (F) receiver-operating characteristic curve of SKA3 expression in KIRP; (G) heat map of SKA3 and its top ten positively or negatively correlated genes; (H) relationship between SKA3 expression and overall survival in KIRP; (I) relationship between SKA3 expression and disease-specific survival in KIRP; (J) relationship between SKA3 expression and progression-free survivacant mark: ${ }^{\text {ns }} P \geq 0.05 ; * P<<0.05 ; * * P<0.01 ; * * * P<0.001$.

tumorigenesis through regulating downstream target-gene expression or binding with other antioncogenes of its family that are involved in apoptosis and repairing damaged DNA, thus promoting tumor-cell growth. ${ }^{31-34}$ Studies have shown that CDK2 overexpression is closely associated with the formation and development of tumors. ${ }^{9,35,36}$ Furthermore, DNA damage can increase p53-protein levels and CDK2 can also phosphorylate p53 and activate downstream signaltransduction pathways. ${ }^{9,37}$ We also found a positive correlation between SKA3 mRNA expression and TP53 $(r=0.370$, 

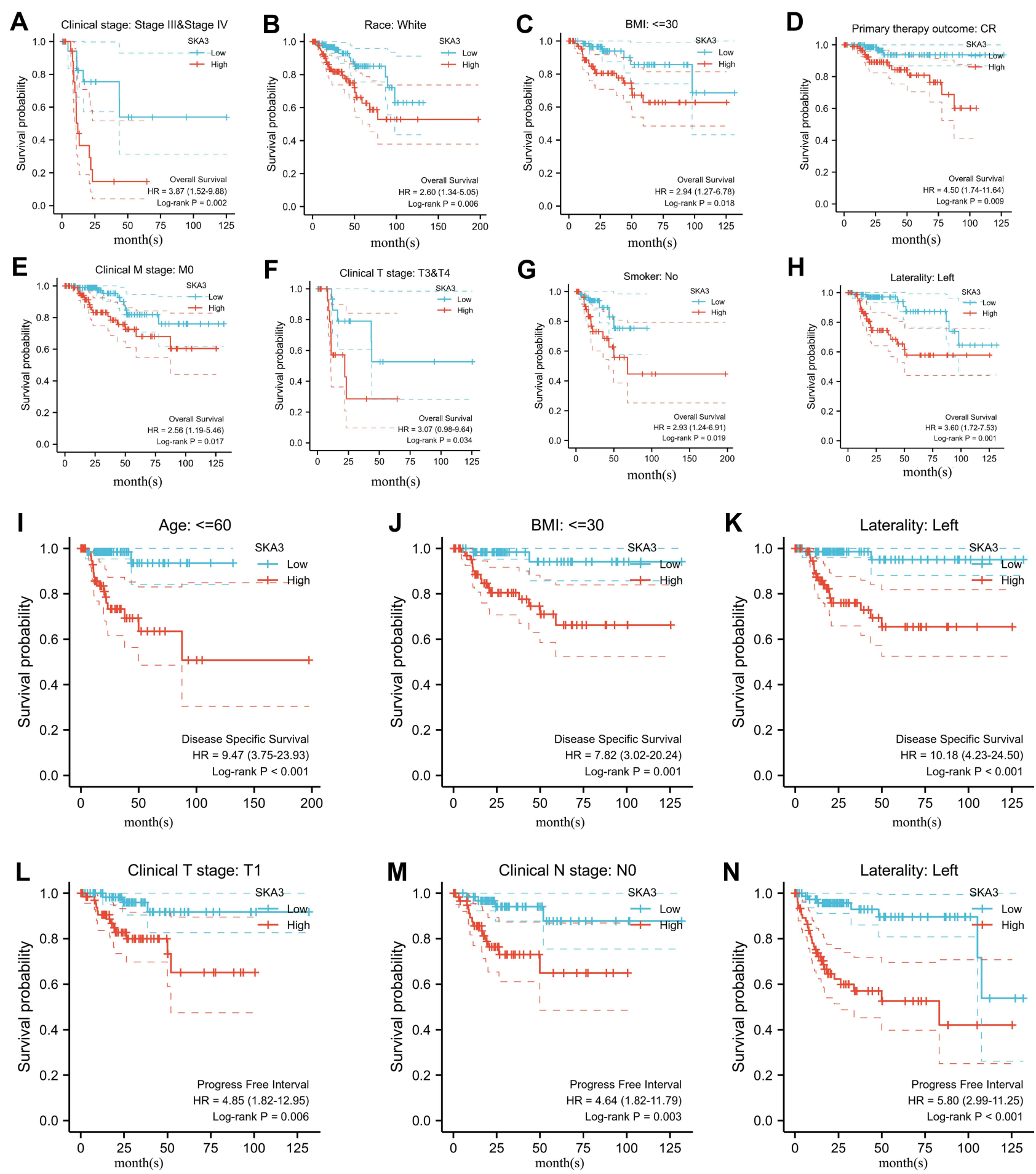

Figure 2 Subgroup analysis of SKA3 expression and survival. (A) Relationship between SKA3 expression and overall survival in stage III and IV; (B) relationship between SKA3 expression and overall survival in white; (C) relationship between SKA3 expression and overall survival in patients with BMI $\leq 30$; (D) relationship between SKA3 expression and overall survival in patients with complete remission (CR); (E) relationship between SKA3 expression and overall survival in patients with stage M0; $(\mathbf{F})$ relationship between SKA3 expression and overall survival in patients with clinical T3-T4; (G) relationship between SKA3 expression and overall survival in nonsmokers; (H) relationship between SKA3 expression and overall survival in left KIRP; (I) relationship between SKA3 expression and disease-specific survival in patients aged $\leq 60$; (J) relationship between SKA3 expression and disease-specific survival in patients with BMI $\leq 30$; (K) relationship between SKA3 expression and disease-specific survival in left KIRP; (L) relationship between SKA3 expression and progression-free survival in patients with TI stage; (M) relationship between SKA3 expression and progression-free survival in patients with N0 stage; (N) relationship between SKA3 expression and progression-free survival in left KIRP. 


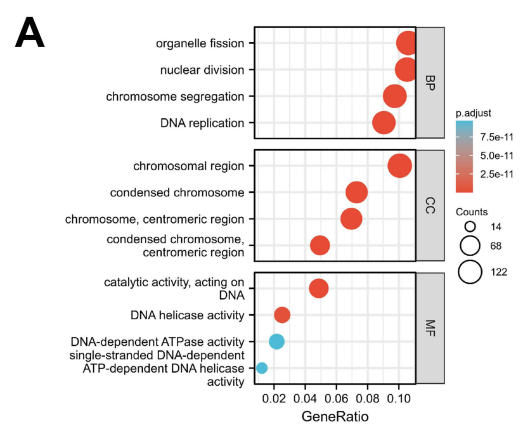

D

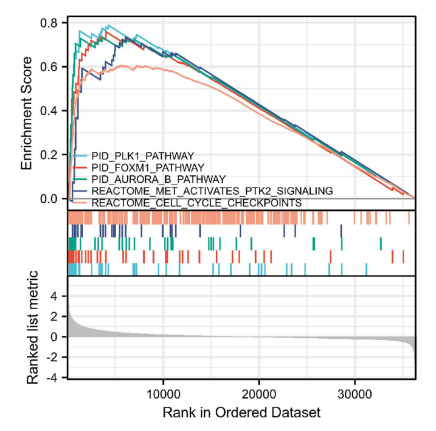

G

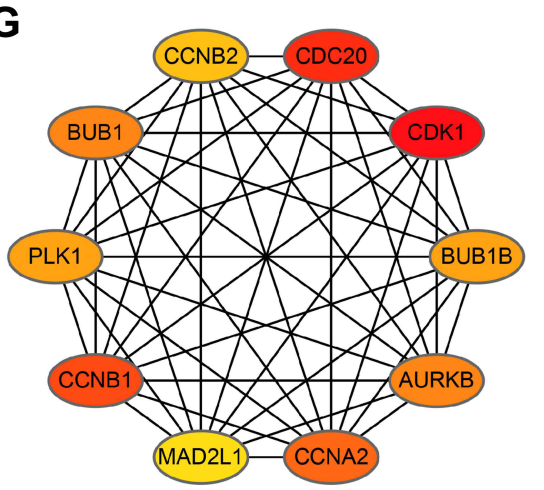

J Survival between high and low GSVA score group

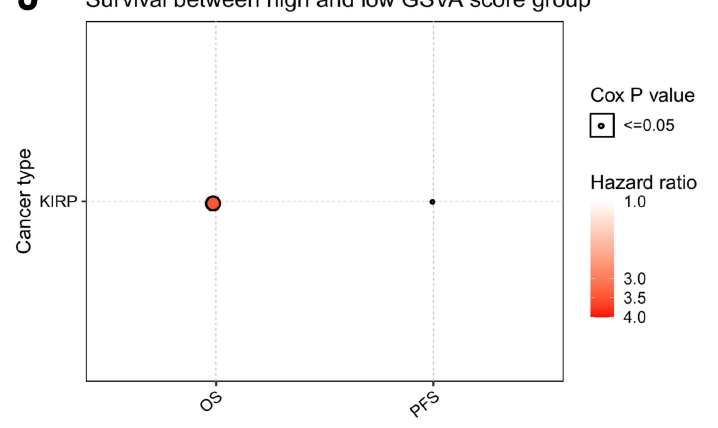

B

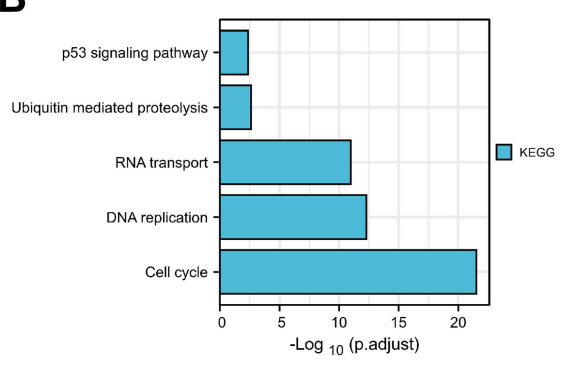

E

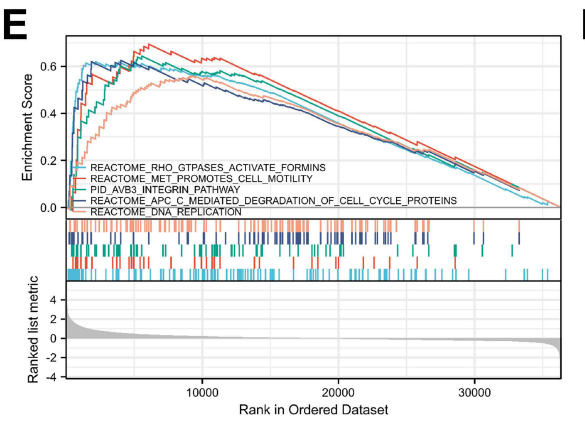

H

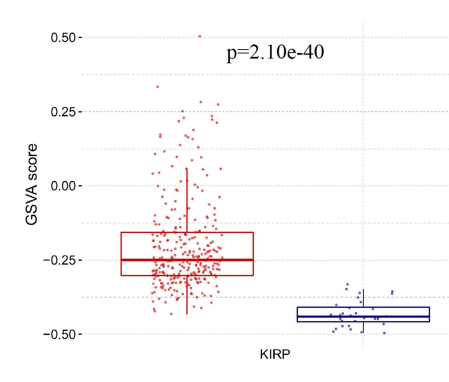

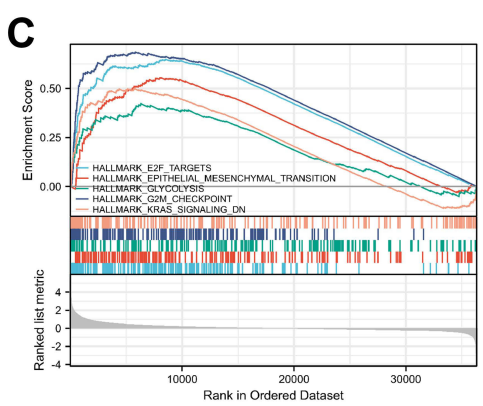

$\mathbf{F}$

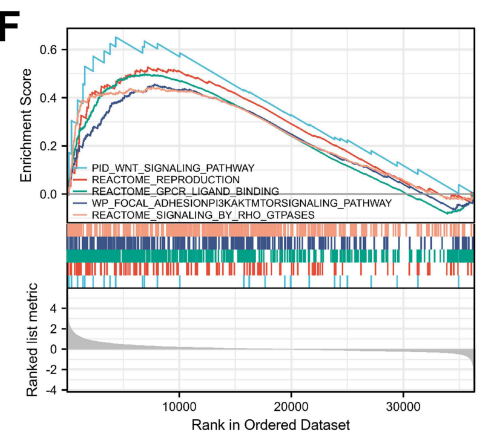

I

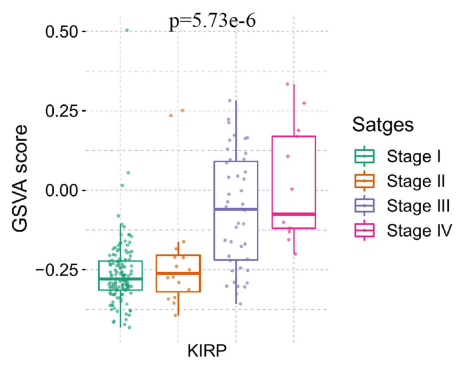

Figure 3 Functional enrichment analysis and the top ten hub genes through protein-protein interaction network. (A) Results of Gene Ontology analysis; (B) results of Kyoto Encyclopedia of Genes and Genomes analysis; (C-F) results of gene -set enrichment analysis; (G) top ten hub genes through protein-protein interaction network; (H) box plot comparing GSVA scores of tumor and normal samples in KIRP; (I) box plot comparing GSVA scores among stages in KIRP; (J) survival difference between GSVA-score groups in KIRP; (K) association between GSVA score and activity of cancer-related pathways in KIRP. Significance defined as $P \leq 0.05$ and false-discovery rate $\leq 0.05$.

$P<0.001)$. Therefore, SKA3-CDK2/p53 might be a possible pathway contributing to oncogenesis and progression of KIRP and deserves to be further validated. The other enriched pathways, including PLK $1,{ }^{8,38}$ Aurora B, ${ }^{39}$ Wnt signaling, ${ }^{10,40}$ and

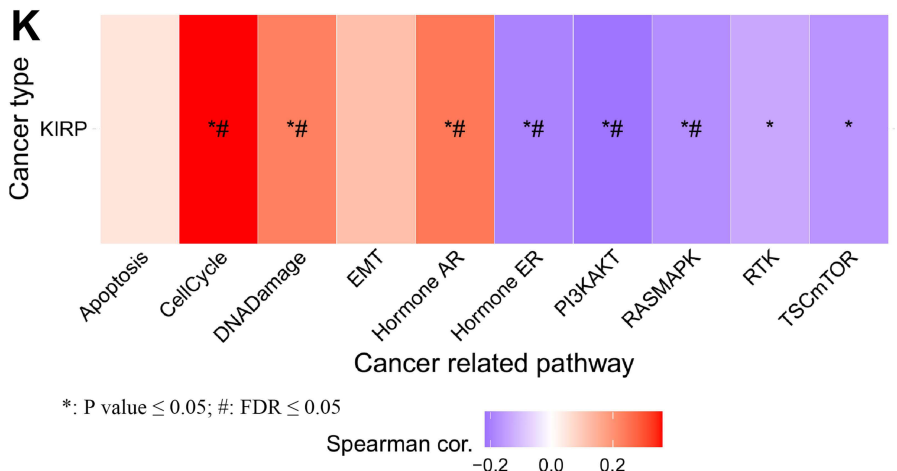



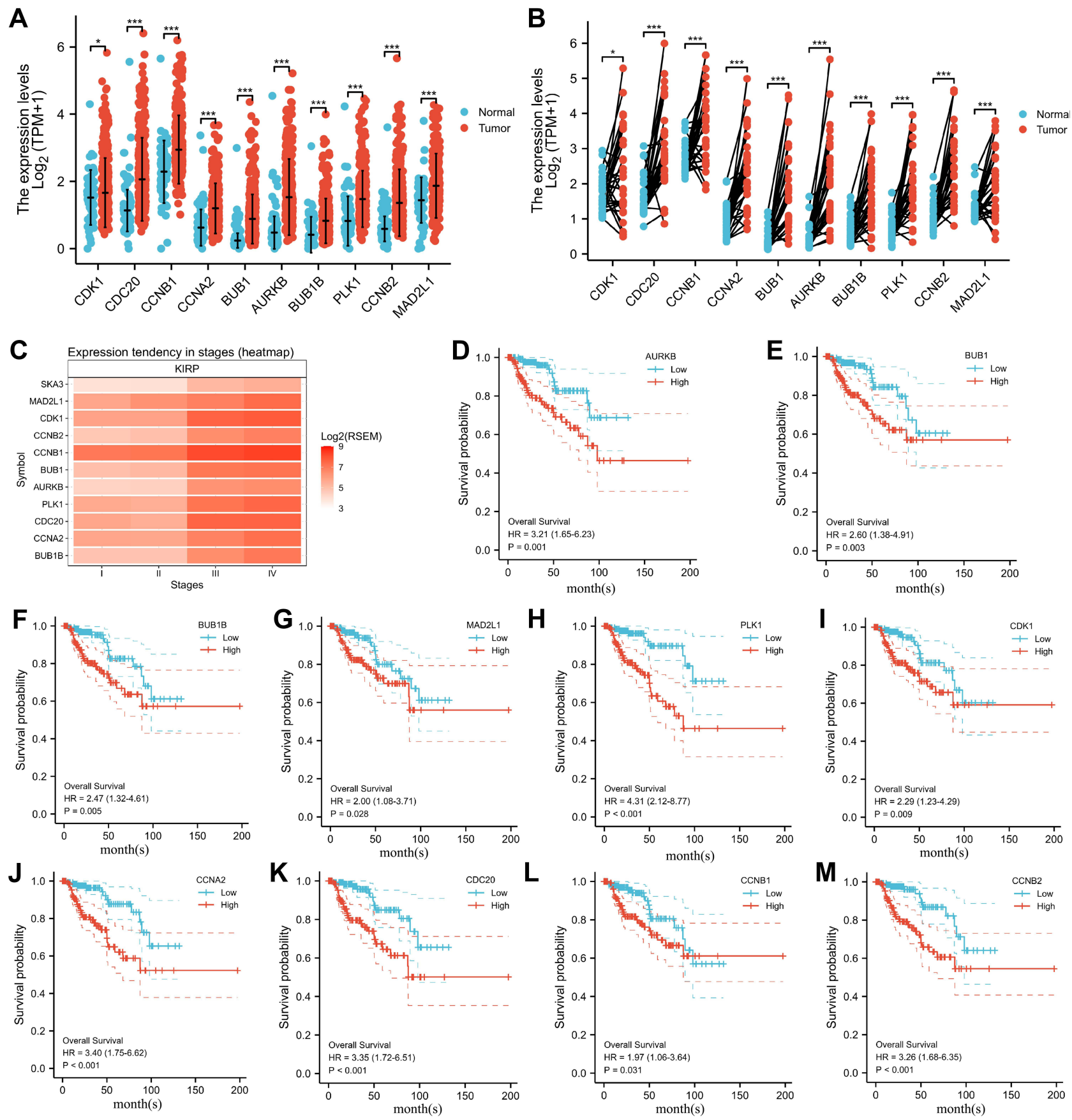

Figure 4 Differential expression of the top ten hub genes and their relationship to prognosis in KIRP patients. (A) Expression in unpaired samples in KIRP; (B) expression in paired samples in KIRP; (C) heat map of gene mRNA-expression profile among stages in KIRP; (D) relationship between AURKB expression and overall survival in KIRP; (E) relationship between $B U B I$ expression and overall survival in KIRP; (F) relationship between BUBIB expression and overall survival in KIRP; (G) relationship between MAD2LI expression and overall survival in KIRP; $(\mathbf{H})$ relationship between PLKI expression and overall survival in KIRP; (I) relationship between CDKI expression and overall survival in KIRP; (J) relationship between CCNA2 expression and overall survival in KIRP; (K) relationship between CDC20 expression and overall survival in KIRP; (L) relationship between $C C N B I$ expression and overall survival in KIRP; (M) relationship between CCNB2 expression and overall survival in KIRP. *P<<0.05; ***P<0.00I.

a series of biological changes that induce tumorigenesis and are associated with resistance to chemotherapy, radiation therapy, drug therapy, and immunotherapy. ${ }^{45} \mathrm{We}$ found that altered SKA3 was positively associated with the namely Buffa, Ragnum, and Winter hypoxia scores.
Therefore, identification of hypoxia involving SKA3 carcinogenesis is also needed. We found that MEK inhibitors, ie, trametinib, selumetinib, PD0325901, and RDEA119, may be sensitive in KIRP patients with high expression of SKA3 and coexpressed genes. This discovery needs 

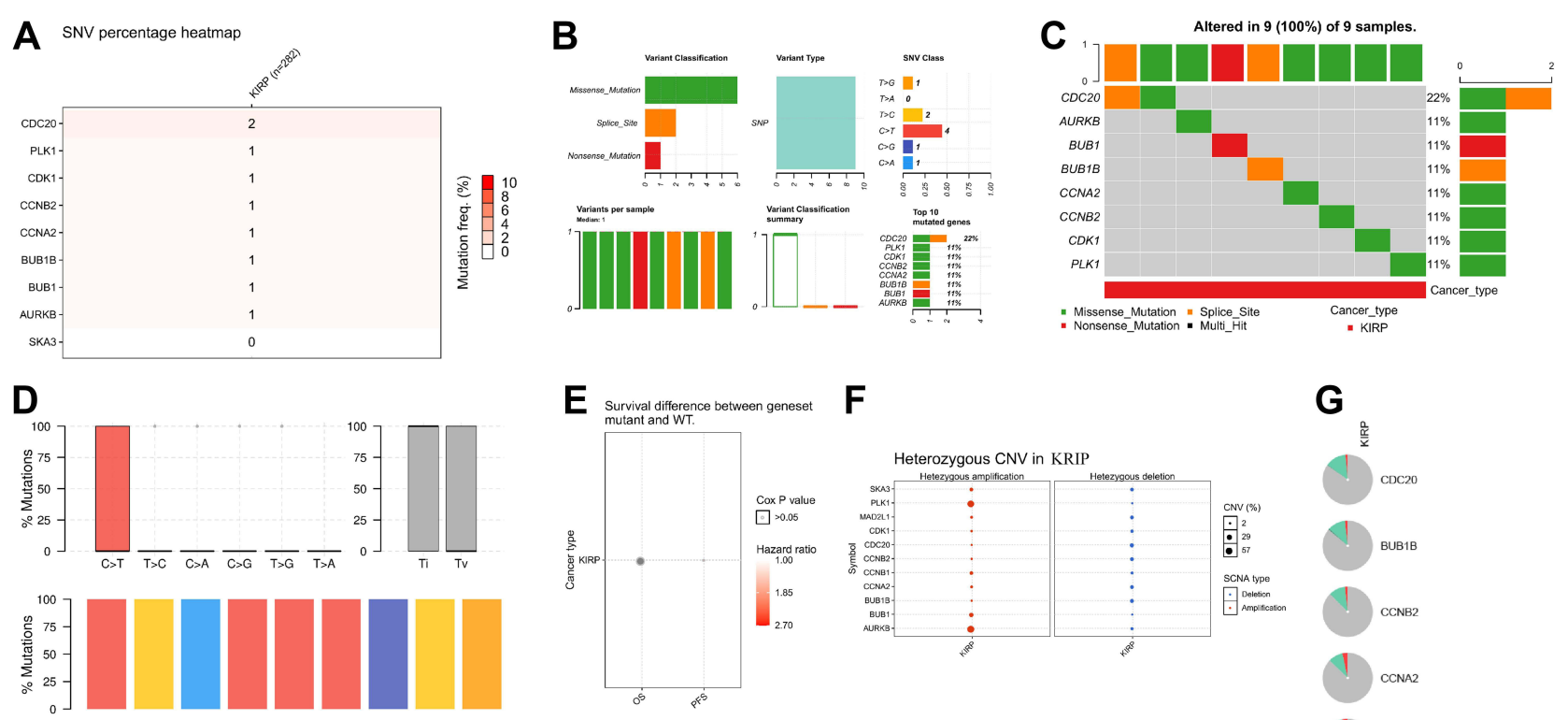

F

G
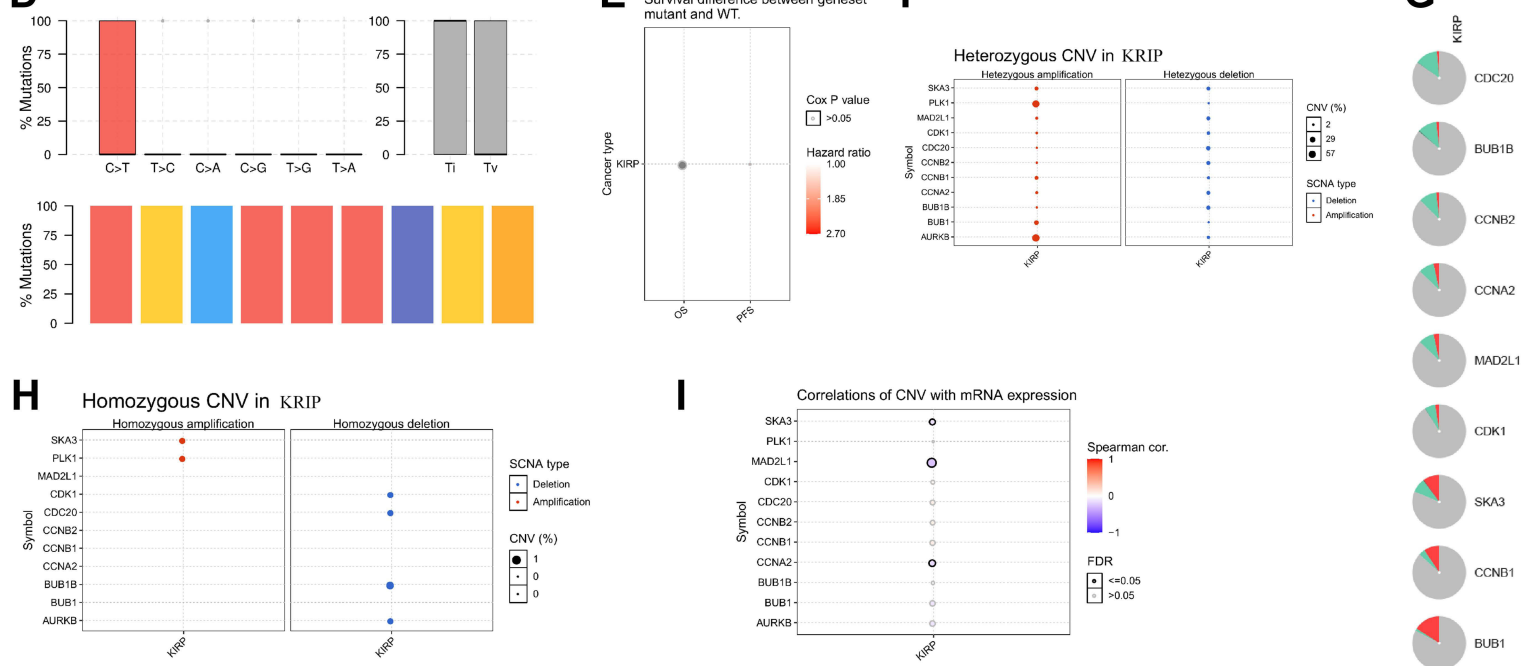

J

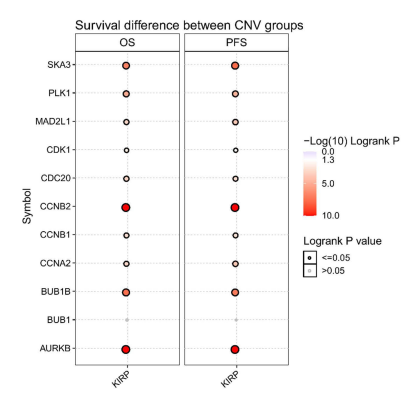

K

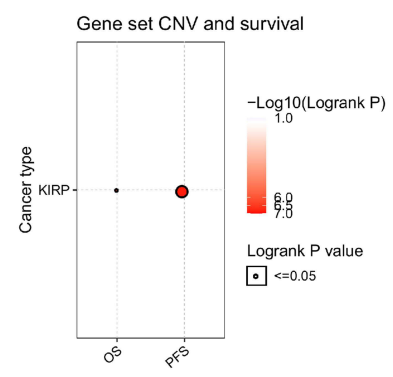

L
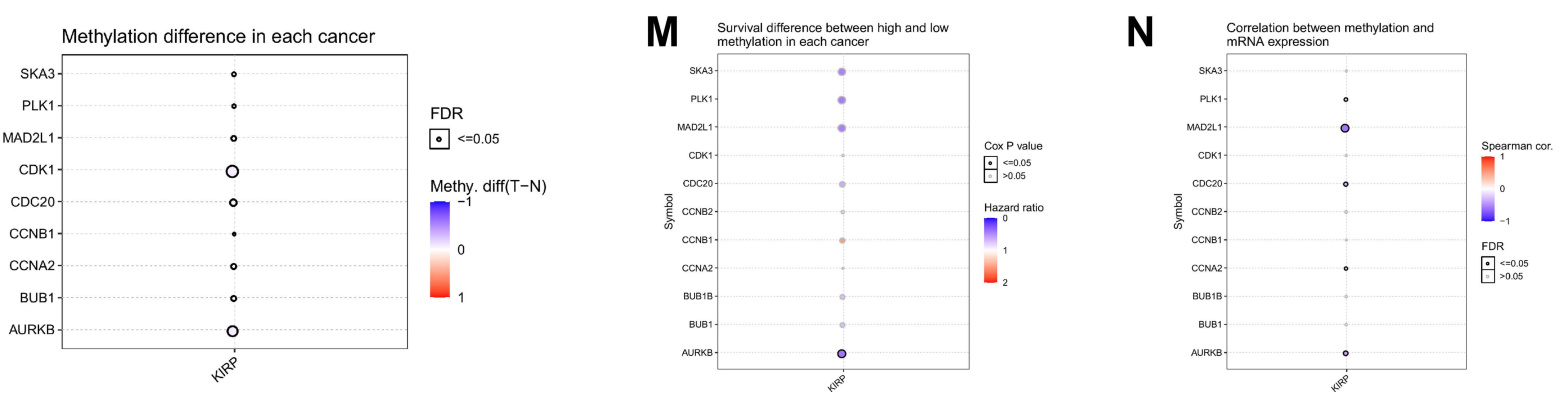

Figure 5 Genetic altion of SKA3 and coexpressed genes in KIRP patients. (A) SNV of SKA3 and hub genes in KIRP; (B) SNV classes of hub-gene set in KIRP; (C) SNV of top ten mutated genes in gene set in KIRP; (D) transition (Ti) and transversion (Tv) classification of SNV of SKA3 gene set and hub genes in KIRP; (E) survival difference between gene-set mutant and wide type; (F) heterozygous CNV of SKA3 gene set and top ten hub genes in KIRP; (G) pie plot summarizing CNV of SKA3 and top ten hub genes in KIRP; $(\mathbf{H})$ homozygous CNV of SKA3 gene set and top ten hub genes in KIRP; (I) correlation of CNV with mRNA expression of SKA3 and top ten hub genes; (J) differences in survival between CNV and wild type in KIRP; (K) survival among gene-set CNV groups in KIRP; (L) methylation differences among tumor and normal samples of SKA3 and top ten hub genes in KIRP; (M) overall survival differences between high- and lowmethylation groups in KIRP; (N) correlations between methylation and mRNA expression of SKA3 and top ten hub genes in KIRP. 

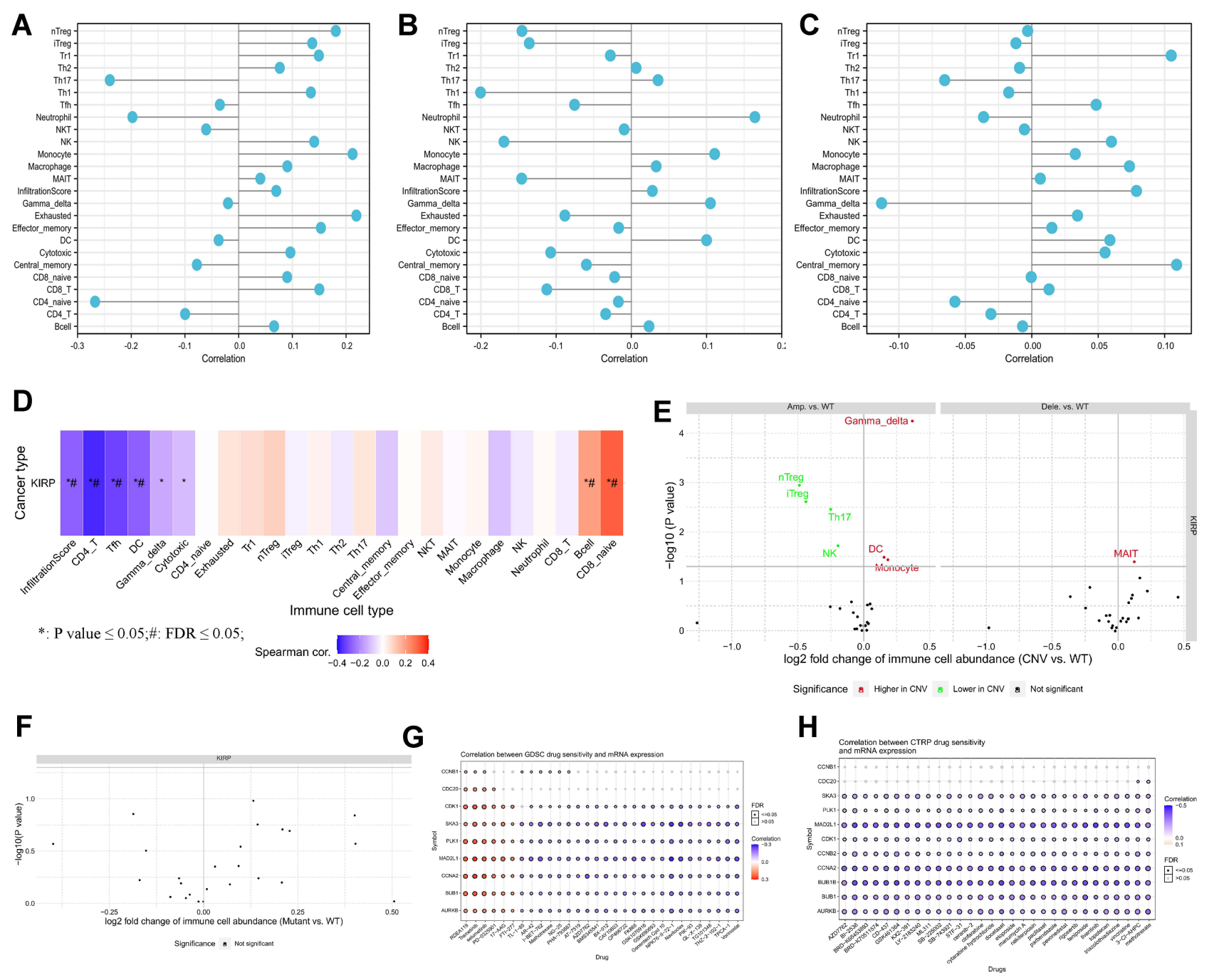

I

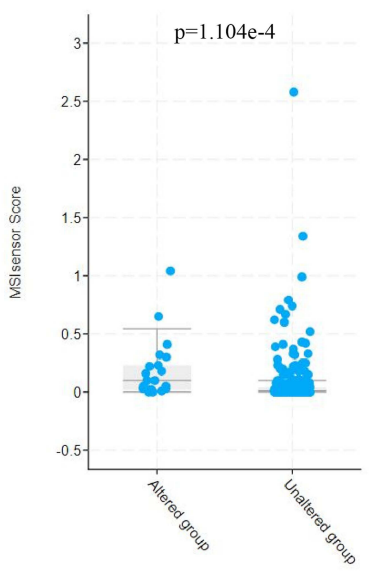

Group
J

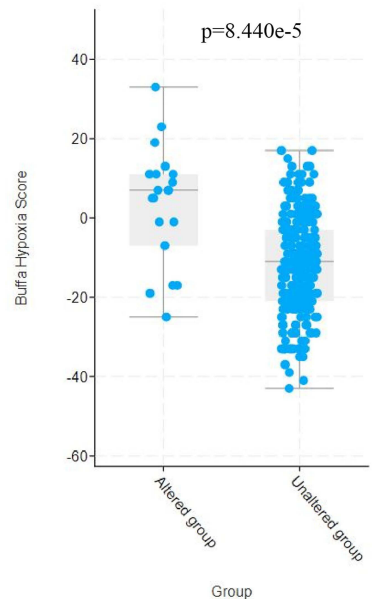

K

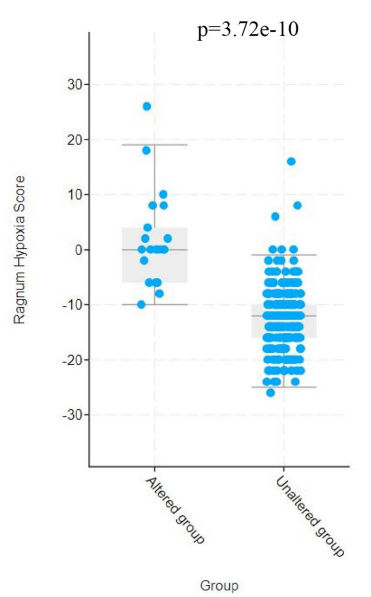

L

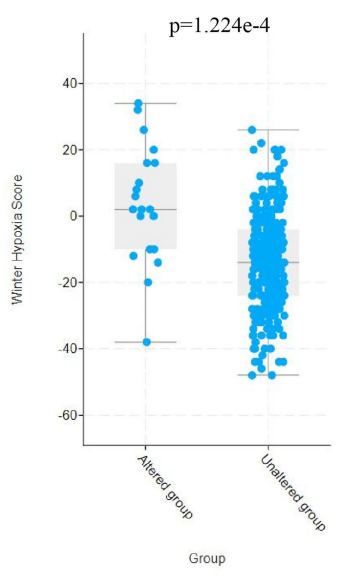

Figure 6 The immunoinfiltration and drug sensitivity of SKA3 and coexpressed genes in KIRP patients and correlation of SKA3 expression and hypoxia-related parameters. (A) association between SKA3 mRNA expression and immunoinfiltration; (B) association between SKA3 CNV and immunoinfiltration; (C) association between SKA3 methylation and immunoinfiltration; (D) associations between SKA3 and top ten hub genes and immunoinfiltration (E) differences in immunoinfiltration between gene-set CNV and wild type (WT); (F) differences in immunoinfiltration among gene-set SNV groups; (G) correlations between gene expression and sensitivity to GDSC drugs (top 30); (H) correlation between gene expression and sensitivity to CTRP drugs (top 30); (I) MSlsensor-score differences of SKA3-altered group and -unaltered group; (J) differences in Buffa hypoxia scores between SKA3-altered group and -unaltered group; (K) differences in Ragnum hypoxia scores between SKA3-altered group and -unalter ed group; (L) differences in Winter hypoxia scores between SKA3-altered group and -unaltered group. 
further verification. Our study did have some limitations: validation using the clinical samples was absent, and further verification of SKA3 function in vitro and in vivo was needed. Further research determining the relationship between SKA3 expression and KIRP type is warranted to help in achieving more precisely personalized treatment.

\section{Conclusion}

SKA3 might contribute to poor prognosis of KIRP through the cell cycle, DNA damage, hormone androgen receptor, hormone estrogen receptor, PI3K/Akt, and Ras/MAPK. SKA3 potentially serves as a prognostic biomarker and target for KIRP.

\section{Ethics}

Data are from the literature and public databases. As such, approval from the West China Hospital of Sichuan University Biomedical Research Ethics Committee was not required. The authors are accountable for all aspects of the work in ensuring that questions related to the accuracy or integrity of any part of the work are appropriately investigated and resolved.

\section{Acknowledgments}

The results shown here are in whole or part based upon data generated by TCGA (https://www.cancer.gov/tcga). Dechao Feng, Facai Zhang, and Ling Liu contributed equally to this work, and should be considered co-first authors. Zhenghua Liu and Lu Yang are co-corresponding authors.

\section{Funding}

This program was supported by the National Natural Science Foundation of China (grant nos. 81974099, 82170785, 81974098, and 82170784), programs from Science and Technology Department of Sichuan Province (grant no. 21GJHZ0246), Young Investigator Award of Sichuan University 2017 (grant no. 2017SCU04A17), Technology Innovation Research and Development Project of Chengdu Science and Technology Bureau (2019-YF05-00296-SN), and the Sichuan UniversityPanzhihua science and technology cooperation special fund (2020CDPZH-4). The funders had no role in study design, data collection or analysis, preparation of the manuscript, or the decision to publish.

\section{Disclosure}

The authors have no conflicts of interest related to this work to declare.

\section{References}

1. Sung H, Ferlay J, Siegel RL, et al. Global cancer statistics 2020: GLOBOCAN estimates of incidence and mortality worldwide for 36 cancers in 185 countries. CA Cancer J Clin. 2021;71(3):209-249. doi:10.3322/caac. 21660

2. Capitanio U, Bensalah K, Bex A, et al. Epidemiology of renal cell carcinoma. Eur Urol. 2019;75(1):74-84. doi:10.1016/j. eururo.2018.08.036

3. Courthod G, Tucci M, Di Maio M, et al. Papillary renal cell carcinoma: a review of the current therapeutic landscape. Crit Rev Oncol Hematol. 2015;96(1):100-112. doi:10.1016/j.critrevonc.2015.05.008

4. Gago-Dominiguez M, Yuan JM, Castelao JE, et al. Family history and risk of renal cell carcinoma. Cancer Epidemiol Biomark Prev. 2011;10:1001-1004.

5. Tannir NM, Jonasch E, Albiges L, et al. everolimus versus sunitinib prospective evaluation in metastatic non-clear cell renal cell carcinoma (ESPN): a randomized multicenter phase 2 trial. Eur Urol. 2016;69(5):866-874. doi:10.1016/j.eururo.2015.10.049

6. Armstrong AJ, Halabi S, Eisen T, et al. Everolimus versus sunitinib for patients with metastatic non-clear cell renal cell carcinoma (ASPEN): a multicentre, open-label, randomised phase 2 trial. Lancet Oncol. 2016;17(3):378-388. doi:10.1016/S1470-2045(15) 00515-X

7. Foley EA, Kapoor TM. Microtubule attachment and spindle assembly checkpoint signalling at the kinetochore. Nat Rev Mol Cell Biol. 2013;14(1):25-37. doi:10.1038/nrm3494

8. Gao W, Zhang Y, Luo H, et al. Targeting SKA3 suppresses the proliferation and chemoresistance of laryngeal squamous cell carcinoma via impairing PLK1-AKT axis-mediated glycolysis. Cell Death Dis. 2020;11(10):919. doi:10.1038/s41419-020-03104-6

9. Hou Y, Wang Z, Huang S, et al. SKA3 promotes tumor growth by regulating CDK2/P53 phosphorylation in hepatocellular carcinoma. Cell Death Dis. 2019;10(12):929. doi:10.1038/ s41419-019-2163-3

10. Zhang J, Liu Y, Pu S, et al. Spindle and kinetochore-associated complex subunit 3 accelerates breast cancer cell proliferation and invasion through the regulation of Akt/Wnt/ $\beta$-catenin signaling. Breast Cancer Res Treat. 2021;186(1):247-258. doi:10.1007/ s10549-020-06078-3

11. Hu DD, Chen HL, Lou LM, et al. SKA3 promotes lung adenocarcinoma metastasis through the EGFR-PI3K-Akt axis. Biosci Rep. 2020;40(2):BSR20194335. doi:10.1042/BSR20194335

12. Goldman MJ, Craft B, Hastie M, et al. Visualizing and interpreting cancer genomics data via the Xena platform. Nat Biotechnol. 2020;38 (6):675-678. doi:10.1038/s41587-020-0546-8

13. Vivian J, Rao AA, Nothaft FA, et al. Toil enables reproducible, open source, big biomedical data analyses. Nat Biotechnol. 2017;35 (4):314-316. doi:10.1038/nbt.3772

14. Liu J, Lichtenberg T, Hoadley KA, et al. An integrated TCGA pan-cancer clinical data resource to drive high-quality survival outcome analytics. Cell. 2018;173(2):400-416.e11.

15. Yu G, Wang LG, Han Y, et al. clusterProfiler: an R package for comparing biological themes among gene clusters. Omics. 2012;16 (5):284-287. doi:10.1089/omi.2011.0118

16. Shannon P, Markiel A, Ozier O, et al. Cytoscape: a software environment for integrated models of biomolecular interaction networks. Genome Res. 2003;13(11):2498-2504. doi:10.1101/gr.1239303

17. Liu CJ, Hu FF, Xia MX, et al. GSCALite: a web server for gene set cancer analysis. Bioinformatics. 2018;34(21):3771-3772. doi:10.1093/bioinformatics/bty411

18. Cerami E, Gao J, Dogrusoz U, et al. The cBio cancer genomics portal: an open platform for exploring multidimensional cancer genomics data. Cancer Discov. 2012;2(5):401-404. doi:10.1158/21598290.CD-12-0095 
19. Gao J, Aksoy BA, Dogrusoz U, et al. Integrative analysis of complex cancer genomics and clinical profiles using the cBioPortal. Sci Signal. 2013;6(269):pl1. doi:10.1126/scisignal.2004088

20. Wang Z, Song Q, Yang Z, et al. Construction of immune-related risk signature for kidney renal papillary cell carcinoma. Cancer Med. 2019;8(1):289-304. doi:10.1002/cam4.1905

21. Cancer Genome Atlas Research Network. Comprehensive molecular characterization of papillary renal-cell carcinoma. $N$ Engl J Med. 2016;374(2):135-145. doi:10.1056/NEJMoa1505917

22. Welburn JP, Grishchuk EL, Backer CB, et al. The human kinetochore Ska1 complex facilitates microtubule depolymerization-coupled motility. Dev Cell. 2009;16(3):374-385. doi:10.1016/j. devcel.2009.01.011

23. Gaitanos TN, Santamaria A, Jeyaprakash AA, et al. Stable kinetochore-microtubule interactions depend on the Ska complex and its new component Ska3/C13Orf3. EMBO J. 2009;28 (10):1442-1452. doi:10.1038/emboj.2009.96

24. Cheeseman IM, Niessen S, Anderson S, et al. A conserved protein network controls assembly of the outer kinetochore and its ability to sustain tension. Genes Dev. 2004;18(18):2255-2268. doi:10.1101/ gad.1234104

25. Zhang Q, Sivakumar S, Chen Y, et al. Ska3 phosphorylated by Cdk1 binds Ndc80 and recruits Ska to kinetochores to promote mitotic progression. Curr Biol. 2017;27(10):1477-1484.e4. doi:10.1016/j. cub.2017.03.060

26. Theis M, Paszkowski-Rogacz M, Buchholz F. SKAnking with Ska3: essential role of Ska3 in cell division revealed by combined phenotypic profiling. Cell Cycle. 2009;8:3435-3437. doi:10.4161/ cc.8.21.9813

27. Daum JR, Wren JD, Daniel JJ, et al. Ska3 is required for spindle checkpoint silencing and the maintenance of chromosome cohesion in mitosis. Curr Biol. 2009;19(17):1467-1472. doi:10.1016/j. cub.2009.07.017

28. Li T, Kon N, Jiang L, et al. Tumor suppression in the absence of p53-mediated cell-cycle arrest, apoptosis, and senescence. Cell. 2012;149(6):1269-1283. doi:10.1016/j.cell.2012.04.026

29. Hager $\mathrm{KM}, \mathrm{Gu}$ W. Understanding the non-canonical pathways involved in p53-mediated tumor suppression. Carcinogenesis. 2014;35(4):740-746. doi:10.1093/carcin/bgt487

30. Bieging KT, Mello SS, Attardi LD. Unravelling mechanisms of p53-mediated tumour suppression. Nat Rev Cancer. 2014;14 (5):359-370. doi:10.1038/nrc3711

31. Wei CL, Wu Q, Vega VB, et al. A global map of p53 transcription-factor binding sites in the human genome. Cell. 2006;124(1):207-219. doi:10.1016/j.cell.2005.10.043
32. Strano S, Fontemaggi G, Costanzo A, et al. Physical interaction with human tumor-derived p53 mutants inhibits p63 activities. J Biol Chem. 2002;277(21):18817-18826. doi:10.1074/jbc.M201405200

33. Di Como CJ, Gaiddon C, Prives C. p73 function is inhibited by tumor-derived p53 mutants in mammalian cells. Mol Cell Biol. 1999;19(2):1438-1449. doi:10.1128/MCB.19.2.1438

34. Schärer E, Iggo R. Mammalian p53 can function as a transcription factor in yeast. Nucleic Acids Res. 1992;20(7):1539-1545. doi:10.1093/nar/20.7.1539

35. Wright RH, Castellano G, Bonet J, et al. CDK2-dependent activation of PARP-1 is required for hormonal gene regulation in breast cancer cells. Genes Dev. 2012;26(17):1972-1983. doi:10.1101/ gad.193193.112

36. Li KK, Ng IO, Fan ST, et al. Activation of cyclin-dependent kinases CDC2 and CDK2 in hepatocellular carcinoma. Liver. 2002;22 (3):259-268. doi:10.1046/j.0106-9543.2002.01629.x

37. Price BD, Hughes-Davies L, Park SJ. Cdk2 kinase phosphorylates serine 315 of human p53 in vitro. Oncogene. 1995;11(1):73-80.

38. Ruan LW, Li PP, Jin LP. SKA3 promotes cell growth in breast cancer by inhibiting PLK-1 protein degradation. Technol Cancer Res Treat. 2020;19:1533033820947488. doi:10.1177/1533033820947488

39. Chan YW, Jeyaprakash AA, Nigg EA, et al. Aurora B controls kinetochore-microtubule attachments by inhibiting Ska complex-KMN network interaction. J Cell Biol. 2012;196 (5):563-571. doi:10.1083/jcb.201109001

40. Li C, Yang J, Lei S, et al. SKA3 promotes glioblastoma proliferation and invasion by enhancing the activation of $\mathrm{Wnt} / \beta$-catenin signaling via modulation of the Akt/GSK-3 $\beta$ axis. Brain Res. 2021;1765:147500. doi:10.1016/j.brainres.2021.147500

41. Hu R, Wang MQ, Niu WB, et al. SKA3 promotes cell proliferation and migration in cervical cancer by activating the PI3K/Akt signaling pathway. Cancer Cell Int. 2018;18:183. doi:10.1186/s12935-0180670-4

42. Liang Y, Zheng Y, Mou K, et al. Inhibition of spindle and kinetochore associated complex subunit 3 suppresses the proliferation and invasion and induced the apoptosis of cutaneous melanoma by affecting the PI3K/Akt pathway. J Biochem Mol Toxicol. 2021;2021:e22895.

43. Zhang Q, Huang $\mathrm{R}, \mathrm{Hu} \mathrm{H}$, et al. Integrative analysis of hypoxia-associated signature in pan-cancer. iScience. 2020;23 (9):101460. doi:10.1016/j.isci.2020.101460

44. Bhandari V, Li CH, Bristow RG, et al. Divergent mutational processes distinguish hypoxic and normoxic tumours. Nat Commun. 2020;11(1):737. doi:10.1038/s41467-019-14052-X

45. Ye Y, Hu Q, Chen H, et al. Characterization of hypoxia-associated molecular features to aid hypoxia-targeted therapy. Nat Metab. 2019;1(4):431-444. doi:10.1038/s42255-019-0045-8
International Journal of General Medicine

\section{Publish your work in this journal}

The International Journal of General Medicine is an international, peer-reviewed open-access journal that focuses on general and internal medicine, pathogenesis, epidemiology, diagnosis, monitoring and treatment protocols. The journal is characterized by the rapid reporting of reviews, original research and clinical studies across all disease areas. The manuscript management system is completely online and includes a very quick and fair peer-review system, which is all easy to use. Visit http://www.dovepress.com/ testimonials.php to read real quotes from published authors. 